\title{
Reaction theory: Status and perspectives
}

\author{
A.M. Moro ${ }^{1}$ and J. Gómez-CAMACHO ${ }^{1,2}$ \\ ${ }^{1}$ Departamento de FAMN, Universidad de Sevilla, Apartado 1065, \\ 41080 Sevilla, Spain \\ ${ }^{2}$ CNA (U. Sevilla, J. Andalucía, CSIC), Av. Tomas A. Edison 7, \\ 41092 Sevilla, Spain
}

\begin{abstract}
The current status of the reaction theory of nuclear collisions involving weakly-bound exotic nuclei is presented. The problem is addressed within the Continuum Discretized Coupled Channel (CDCC) framework, recalling its foundations and applications, as well as its connection with the Faddeev formalism. Recent developments and improvements of the method, such as core and target excitations and the extension to three-body projectiles, are presented. The use of the CDCC wave function in the calculation of inclusive breakup reactions is also introduced.
\end{abstract}

\section{Introduction}

Ongoing and planned radioactive beam facilities will produce in near future accurate reaction data covering unexplored areas of the nuclear chart. The extraction of meaningful information from these experiments will demand new advances in nuclear structure and reaction theories.

The description of the collision between composite nuclei is a very complicated quantum mechanical many-body problem which, in general, cannot be solved exactly. Although, ideally, the problem could be solved from a many-body viewpoint, starting with some effective nucleon-nucleon interaction, in practice one needs to resort to additional approximations, aimed at making the problem numerically tractable. These approximate models try 
to emphasize specific degrees of freedom, those which are expected to dominate the reaction dynamics, or those in which one is interested, and recast the problem in terms of an effective Hamiltonian in which these selected degrees of freedom appear explicitly.

Within the Coupled Channels (CC) method, the Hamiltonian that describes a scattering problem, in which some internal degrees of freedom (from projectile, target or both) can be excited, is:

$$
H=H_{\mathrm{int}}(\xi)+\hat{T}_{\mathbf{R}}+U(\mathbf{R}, \xi),
$$

with $H_{\text {int }}$ as the internal Hamiltonian, depending on the internal coordinates $\xi, \hat{T}_{\mathbf{R}}$ the kinetic energy operator, and $U$ the projectile-target interaction, depending both on their relative coordinate $\mathbf{R}$ and $\xi$. In CC methods [1], the scattering wave function is written as an expansion of the form

$$
\Psi^{\mathrm{CC}}(\xi, \mathbf{R})=\sum_{\beta} \Phi_{\beta}(\xi) \chi_{\beta}(\mathbf{R}),
$$

where $\Phi_{\beta}(\xi)$ are eigenstates of $H_{\mathrm{int}}$. The index $\beta$ represents as set of quantum numbers characterizing a given projectile or target state. The functions $\chi_{\beta}(\mathbf{R})$ describe the projectile-target relative motion and are obtained by inserting the expansion (2) into the Schrödinger equation, $[H-E] \Psi^{\mathrm{CC}}=0$. This gives rise to a set of coupled differential equations which, solved with the proper boundary conditions, produce the scattering observables. In practice, the $\mathrm{CC}$ method is applied using a partial wave decomposition of the total wave function as explained elsewhere $[1,2]$.

In the following section, we will adapt the general CC method to relevant cases in exotic nuclei scattering. We will identify the relevant internal degrees of freedom $\xi$, and specify the particular expression of the internal wave function $\Phi_{\beta}(\xi)$.

\section{The Continuum Discretized Coupled Channels (CDCC) method}

When dealing with the scattering of a two-body weakly-bound nucleus on a well bound target, an important degree of freedom that must be taken into account is the dissociation (i.e., breakup) of the projectile into its constituents. Within the CC scheme, this problem can be accurately treated using the CDCC method. This method was originally introduced by G. Rawitscher [3] and later refined by the Pittsburgh-Kyushu collaboration [2,4] 

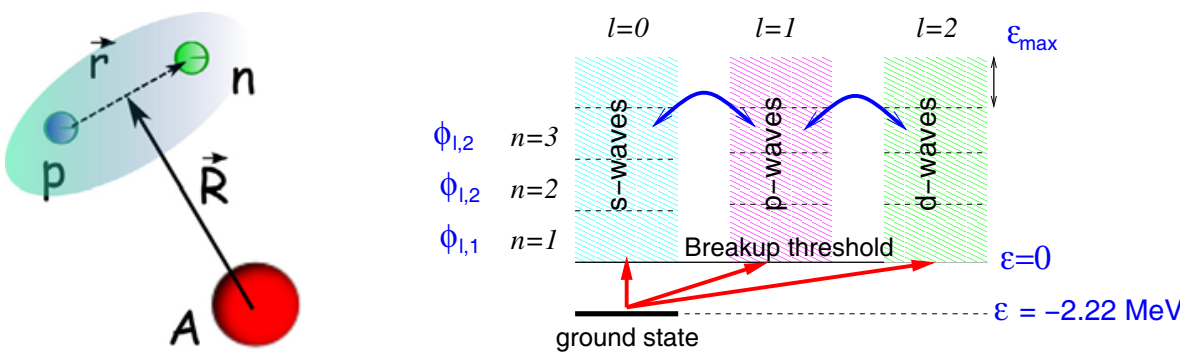

Figure 1: Left: Relevant coordinates for a deuteron-target scattering problem. Right: Illustration of continuum discretization for the same problem.

to describe the effect of the breakup channels on the elastic scattering of deuterons. Denoting the reaction by $a+A$, with $a=c+v$ (referred hereafter as the core and valence particles, respectively), the method assumes the effective three-body Hamiltonian

$$
H=H_{\text {proj }}+\hat{T}_{\mathbf{R}}+U_{c A}\left(\mathbf{r}_{c A}\right)+U_{v A}\left(\mathbf{r}_{v A}\right),
$$

with $H_{\text {proj }}=T_{\mathbf{r}}+V_{v c}$ the projectile internal Hamiltonian, $\hat{T}_{\mathbf{r}}$ and $\hat{T}_{\mathbf{R}}$ are kinetic energy operators, $V_{c v}$ the intercluster interaction and $U_{c A}$ and $U_{v A}$ are the core-target and valence-target optical potentials evaluated at the corresponding incident energy per nucleon. In the CDCC method the three-body wave function of the system is expanded in terms of the eigenstates of the Hamiltonian $H_{\text {proj }}$ including both bound and unbound states. Since the latter form a continuum, a procedure of discretization is applied, consisting in representing this continuum by a finite and discrete set of square-integrable functions. So, in this case, $\xi \equiv \mathbf{r}$, and the internal wave functions in Eq. (2) take the form

$$
\Phi_{\beta}(\xi) \equiv \phi_{n, \ell, j \pi}^{p}\left(k_{n}, \mathbf{r}\right),
$$

where $\phi_{n, \ell, j \pi}^{p}\left(k_{n}, \mathbf{r}\right)$ is a discrete continuum wave function representing a region of the continuum with orbital (total) angular momentum $\ell(j)$ and nominal wave number $k_{n}$.

The standard CDCC method relies on a strict three-body model of the reaction $(c+v+A)$, and has proven to be rather successful to describe elastic and exclusive breakup cross sections of deuterons and other weakly bound two-body nuclei (like ${ }^{6,7} \mathrm{Li}$ ) [2]. However, it has limitations. The assumption of inert bodies is not always justified, since excitations of the projectile constituents $(c$ and $v$ ) and of the target $(A)$ may take place in addition to the projectile dissociation. Furthermore, the two-body picture 
may be inadequate for some nuclei as, for example, in the case of the Borromean systems, i.e., three-body systems in which the binary sub-systems are unbound, such as ${ }^{6} \mathrm{He}$ and ${ }^{11} \mathrm{Li}$. Some extensions of the CDCC method to deal with the these situations are outlined in the following.

\subsection{Inclusion of target excitations}

The explicit inclusion of target excitation was done by the Kyushu group in the early days of the CDCC method [4]. Target excitation arises from the non-central part of the valence-target and core-target interactions and, consequently, the effective Hamiltonian, Eq. (3), must be generalized to:

$$
H=H_{\text {proj }}(\mathbf{r})+H_{\text {tar }}\left(\xi_{t}\right)+\hat{T}_{\mathbf{R}}+U_{c A}\left(\mathbf{r}_{c A}, \xi_{t}\right)+U_{v A}\left(\mathbf{r}_{v A}, \xi_{t}\right),
$$

where $H_{\mathrm{tar}}\left(\xi_{t}\right)$ is the target Hamiltonian, depending on the target degrees of freedom (denoted as $\xi_{t}$ ). Note that $c-A$ and $v-A$ interactions depend now, in addition to the corresponding relative coordinate, on $\xi_{t}$. So, the relevant degrees of freedom are $\xi \equiv\left\{\mathbf{r}, \xi_{t}\right\}$ and the internal states become

$$
\Phi_{\beta}(\xi) \equiv\left[\phi_{n, \ell, j \pi}^{p}\left(k_{n}, \mathbf{r}\right) \otimes \phi_{m}^{t}\left(\xi_{t}\right)\right]_{J M},
$$

which are just products of the target eigenstates times the continuum discretized projectile wave function. They can be coupled to a total internal angular momentum $J, M$.

In the calculations performed in Ref. [4], the target excitation was treated within the vibrational model and the $c-A$ and $v-A$ interactions represented by deformed optical potentials. They performed calculations for the $d+{ }^{58} \mathrm{Ni}$ reaction at $E_{d}=22$ and $80 \mathrm{MeV}$, including $s$-wave breakup of the deuteron and excitation of the target to its first excited state $\left(2^{+}\right)$. In this case, deuteron breakup was found to dominate over target excitation.

Recently, the problem has been also addressed by P. Chau [5] and applied also to deuteron elastic and inelastic scattering, but the method does not seem to have been implemented in publicly available codes.

\subsection{Inclusion of core excitations}

Similarly to the case of target excitation, excitations of the projectile constituents ( $c$ and $v$ in our case) are also possible. For example, in the important case of the scattering of two-body halo nuclei, collective excitations of the core can be important. These core excitations will affect not only the reaction dynamics, but also the structure of the projectile. In the inert-core 
picture, the projectile states will correspond to pure single-particle or cluster states. On the other hand, if the core is allowed to excite, the projectile states will contain in general admixtures of these core excited components. These two effects (dynamic and structure effects) have been recently investigated within extended versions of the DWBA and CDCC methods [6-9]. To this end, the following effective three-body Hamiltonian has been used:

$$
H=H_{\text {proj }}\left(\mathbf{r}, \xi_{c}\right)+\hat{T}_{\mathbf{R}}+U_{c A}\left(\mathbf{r}_{c A}, \xi_{c}\right)+U_{v A}\left(\mathbf{r}_{v A}\right) .
$$

Note that the core degrees of freedom $\left(\xi_{c}\right)$ appear in the projectile Hamiltonian (structure effect) as well as in the core-target interaction (dynamical effect). In the weak coupling limit, the basis functions can be expressed as

$$
\Phi_{\beta}(\xi) \equiv \sum_{\alpha}\left[\varphi_{\alpha}^{p}(\mathbf{r}) \otimes \Phi_{I}\left(\xi_{c}\right)\right]_{J M},
$$

where $\xi \equiv\left\{\xi_{c}, \mathbf{r}\right\}$. The label $\alpha$ denotes the set of quantum numbers $\{\ell, s, j, I\}$, with $I$ the core intrinsic spin, $\vec{j}=\vec{\ell}+\vec{s}$ and $\vec{J}=\vec{j}+\vec{I}$. The functions $\Phi_{I}\left(\xi_{c}\right)$ and $\varphi_{\alpha}^{p}(\mathbf{r})$ describe, respectively, the core states and the valence-core relative motion. For continuum states, a procedure of continuum discretization is used, similar to the case of the standard CDCC.

Once the projectile states (8) have been calculated, the three-body wave function is written as an expansion in terms of these states, according to Eq. (2). Calculations using this extended CDCC method (XCDCC) were first performed by Summers et al. $[8,10]$ for ${ }^{11} \mathrm{Be}$ and ${ }^{17} \mathrm{C}$ on ${ }^{9} \mathrm{Be}$ and ${ }^{11} \mathrm{Be}+p$. Later on, De Diego et al. [9] performed calculations for ${ }^{11} \mathrm{Be}$ on ${ }^{1} \mathrm{H},{ }^{64} \mathrm{Zn}$ and ${ }^{208} \mathrm{~Pb}$. These calculations suggest that, for the proton target case, dynamic core excitations may increase significantly the breakup cross sections whereas, for the heavier targets, the dynamic core excitation mechanism is small but its effect on the structure of the projectile is still important. As an example, we show in Fig. 2 the XCDCC calculations for the reaction ${ }^{11} \mathrm{Be}+p$ at $63.7 \mathrm{MeV} /$ nucleon (adapted from Ref. [9]).

A no-recoil DWBA version of this approach has been also proposed in Refs. [6,7]. Calculations performed using this formalism have confirmed the conclusions of the more elaborated XCDCC calculations and have also served to evidence that the interplay between the single-particle and dynamic core excitation mechanisms produce a distinctive effect on the interference pattern of the resonant breakup angular distributions [11]. 


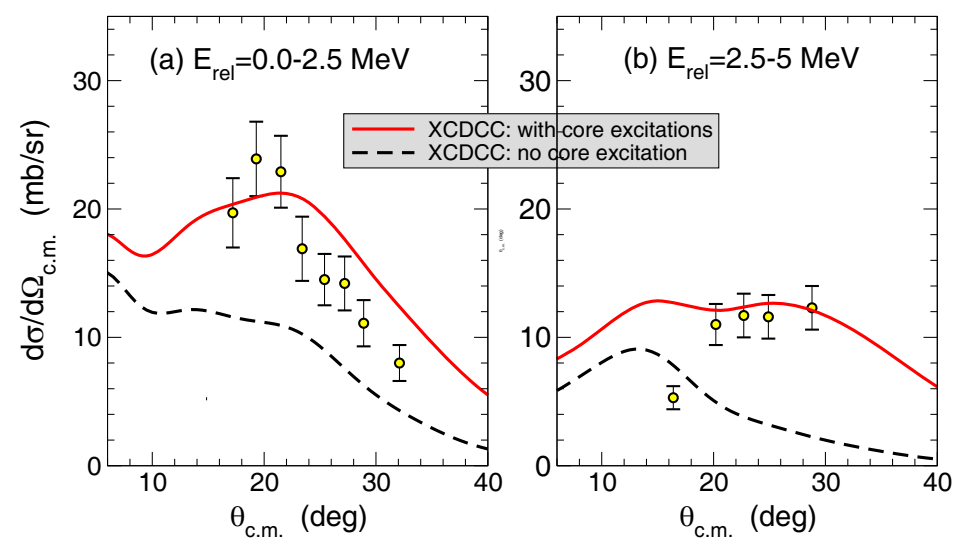

Figure 2: (Color online) Differential breakup cross sections, with respect to the outgoing ${ }^{11} \mathrm{Be}^{*}$ c.m. scattering angle, for the breakup of ${ }^{11} \mathrm{Be}$ on protons at 63.7 $\mathrm{MeV} /$ nucleon. Left and right panels correspond to the neutron-core relative energy intervals $E_{\text {rel }}=0-2.5 \mathrm{MeV}$ and $E_{\mathrm{rel}}=2.5-5 \mathrm{MeV}$, respectively. Adapted from [9].

\subsection{Extension to three-body projectiles}

To study the scattering of three-body projectiles, such as Borromean nuclei, the Hamiltonian (3) must be generalized in order to take into account the three-body structure of the projectile. For example, for a Borromean system with a structure of the form $c+v_{1}+v_{2}$ one may use the Hamiltonian

$$
H=H_{\text {proj }}\left(\mathbf{r}_{1}, \mathbf{r}_{2}\right)+\hat{T}_{\mathbf{R}}+U_{c A}+U_{v_{1} A}+U_{v_{2} A},
$$

where $H_{\text {proj }}\left(\mathbf{r}_{1}, \mathbf{r}_{2}\right)$ is the projectile (three-body) Hamiltonian (depending now on two relative coordinates), and $U_{v_{i}}\left(\mathbf{r}_{v_{i} A}\right)$ the valence-target effective interactions. Clearly, the calculation of the projectile states will be much more involved than in the two-body case. The basis functions can be expressed in several ways. For example, using independent coordinates,

$$
\Phi_{\beta}(\xi)=\sum_{\alpha}\left[\varphi_{\alpha}\left(\mathbf{r}_{1}, \mathbf{r}_{2}\right)\right]_{J M},
$$

where $\xi \equiv\left\{\mathbf{r}_{1}, \mathbf{r}_{2}\right\}$, which are the internal coordinates of the three-body system. The label $\alpha$ denotes the set of quantum numbers $\left\{\ell_{1}, \ell_{2}, K, \ldots\right\}$, required to characterize the three-body state, which may vary depending on the three-body approach used. Note that $\Phi_{\beta}(\xi)$ is an (approximate) eigenstate of the projectile three-body Hamiltonian, with a given energy and angular momentum. 

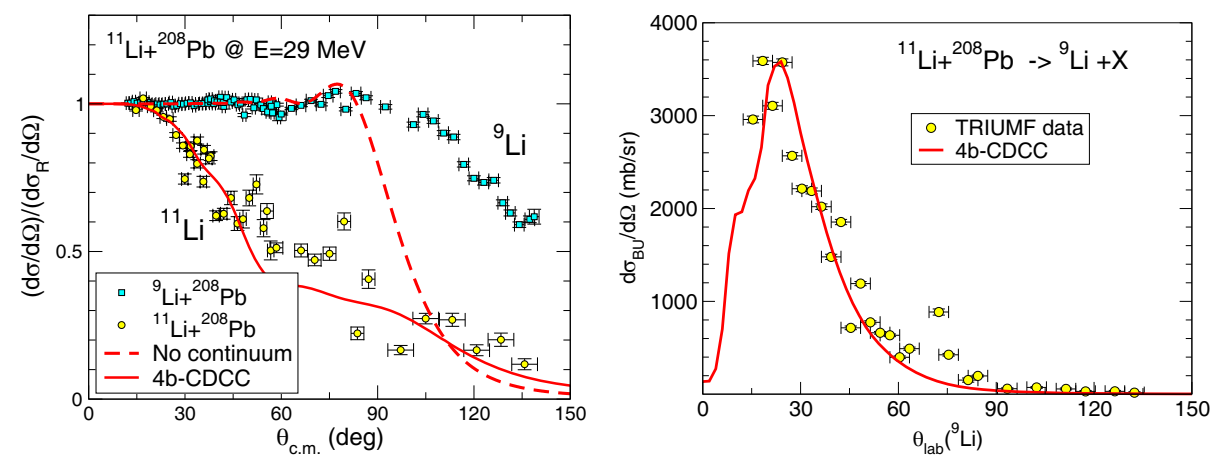

Figure 3: Left: Differential elastic cross section, relative to Rutherford, for the ${ }^{11} \mathrm{Li}+{ }^{208} \mathrm{~Pb}$ reaction at an incident energy of $29 \mathrm{MeV}$. The solid (dashed) line is the four-body CDCC calculation including (excluding) ${ }^{11} \mathrm{Li}$ continuum states. The ${ }^{9} \mathrm{Li}$ elastic scattering data are also included for comparison. Right: Angular distribution of ${ }^{9} \mathrm{Li}$ fragments resulting from the same reaction, compared with the four-body CDCC calculation. The data and calculations are from Refs. [14,15].

The formulation and application of this four-body CDCC method was first done by the groups at Kyushu [12] and Seville [13]. As an application of the method, we show in Fig. 3 the elastic (left) and breakup (right) of ${ }^{11} \mathrm{Li}$ on ${ }^{208} \mathrm{~Pb}$ at $29 \mathrm{MeV}[14,15]$. In both panels, the full four-body CDCC calculations are represented by the solid line.

\section{Comparison with the Faddeev formalism}

The CDCC method was originally devised as a physically sound and numerically appealing ansatz for the three-body wave function, rather than as a formally rigorous solution of the three-body problem. The latter was provided by Faddeev in the 60s [16], who showed that the exact solution of this problem can be obtained by solving a system of coupled-differential equations, named the Faddeev equations after him. The numerical solution of the Faddeev equations is very involved but, recently, it has been possible to solve these equations for a number of situations $[17,18]$, thus providing a valuable benchmark to more approximate models. These comparative studies have shown that the elastic and breakup observables calculated with the CDCC method agree in general very well with the Faddeev solution (see Fig. 4). However, there are also kinematical situations [18] in which the agreement is not good, for example, when the incident energy is too low. This result calls for additional studies and, possibly, for extensions or improvements of the CDCC formalism. 


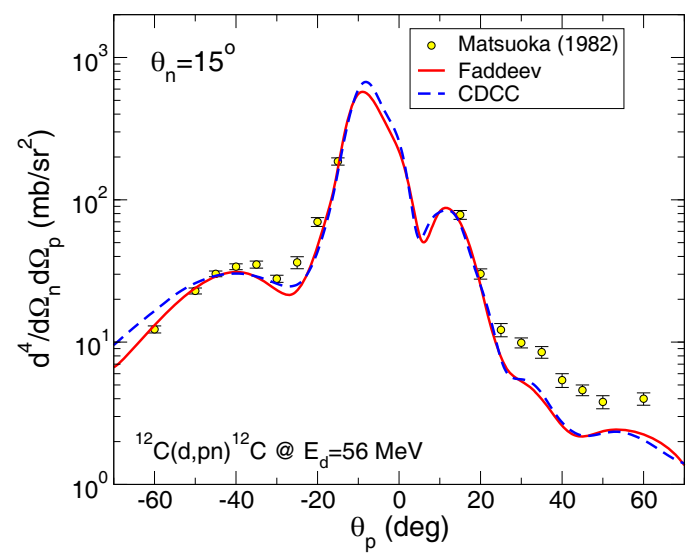

Figure 4: Breakup differential cross section for ${ }^{12} \mathrm{C}(p, p n){ }^{12} \mathrm{C}$ at $E_{d}=56 \mathrm{MeV}$, as a function of the proton scattering angle and for a fixed neutron detection angle of $15^{\circ}$. The Faddeev and CDCC calculations are given by the solid and dashed lines, respectively. The circles are the data from Ref. [19].

\section{The problem of inclusive breakup}

In previous sections, we have considered only exclusive breakup processes in which the three fragments $(c, v$ and $A$ ) survive after the collision and are observed in a definite internal state. In particular, when all fragments end up in their ground state, the process is called elastic breakup (EBU). A qualitatively different scenario occurs when the final state of one or more fragments is not specified in the final state. For example, this is the case of reactions of the form $A(a, c) X$, in which only one of the projectile constituents (the core in this case) is observed. The angular/energy distribution of the $c$ fragments will contain contribution from all possible final states of the $v+A$ system. This includes the EBU channel, in which $v$ and $A$ remain in their ground state, but also $v$ transfer, breakup accompanied by excitations of $A$, and $v+A$ fusion [named incomplete fusion (ICF)]. These non-elastic breakup components (NEB) must be added to the EBU component to give the total inclusive breakup. Whereas the calculation of EBU can be accurately done within the CDCC method and other approaches, the calculation of NEB is more involved due to the large number of accessible states. The problem was addressed in the 1980s by several groups, which developed formal techniques to reduce the sum over final states to a closed form. We cite here the theory developed by Ichimura, Austern and Vincent (IAV) $[2,20,21]$, in which the double differential cross section for NEB with 
respect to the angle and energy of the $c$ fragments is given by

$$
\left.\left.\frac{d^{2} \sigma}{d E_{c} d \Omega_{c}}\right|_{\mathrm{NEB}}=-\frac{2}{\hbar v_{i}} \rho_{c}\left(E_{c}\right)\left\langle\varphi_{v}\left(\vec{k}_{c}\right)\left|\operatorname{Im}\left[U_{v A}\right]\right| \varphi_{v}\left(\vec{k}_{c}\right)\right)\right\rangle,
$$

where $\rho_{c}\left(E_{c}\right)=k_{c} \mu_{c} /\left((2 \pi)^{3} \hbar^{2}\right), U_{v A}$ is the optical potential describing $v+$ $A$ elastic scattering, and $\varphi_{v}\left(\vec{k}_{c}, \mathbf{r}_{v A}\right)$ is the wave function describing the evolution of the $v$ particle after dissociating from the projectile, when the core is scattered with momentum $\vec{k}_{c}$ and the target remains in its ground state. This function is obtained as a solution of the inhomogeneous equation

$$
\left(E_{v}^{+}-K_{v}-U_{v A}\right) \varphi_{v}\left(\vec{k}_{c}, \mathbf{r}_{v A}\right)=\left(\chi_{c}^{(-)}\left(\vec{k}_{c}\right)\left|V_{\text {post }}\right| \Psi^{3 b}\right\rangle
$$

where $E_{v}=E-E_{c}, \chi_{c}^{(-)}\left(\vec{k}_{c}, \mathbf{r}_{c B}\right)$ is the distorted-wave describing the scattering of the outgoing $c$ fragment with respect to the $B \equiv v+A$ system, obtained with some optical potential $U_{c B}$, and $V_{\text {post }} \equiv V_{v c}+U_{c A}-U_{c B}$ is the post-form transition operator. This equation is to be solved with outgoing boundary conditions.

Although IAV suggest approximating the three-body wave function appearing in the source term of Eq. (11), $\Psi^{3 b}$, by the CDCC one, a simpler choice is to use the DWBA approximation $\psi_{v}^{3 b} \approx \chi_{a}^{(+)}(\mathbf{R}) \phi_{c v}(\mathbf{r})$, where $\chi_{a}^{(+)}$ is a distorted wave describing $a+A$ elastic scattering, obtained with some optical potential, and $\phi_{c v}$ is the projectile ground state wave function.

This DWBA version of the IAV model has been recently revisited by some groups, and applied to several deuteron $[22,23]$ and ${ }^{6} \mathrm{Li}$ induced reactions [24], showing in most cases a very promising agreement with the existing inclusive data. Further applications and developments are in progress.

\section{Conclusions}

The nuclear reaction theory community has developed in recent years quantum mechanical methods, based on the coupled channels approach, which allow to interpret the exclusive scattering experiments of exotic nuclei. These methods have been compared, in some cases, with exact three-body Faddeev calculations. Preliminary extensions of these methods to the calculation of inclusive breakup cross sections is in progress show very encouraging results.

\section{References}

[1] I.J. Thompson, F.M. Nunes, Nuclear Reactions for Astrophysics, Cambridge, UK: Cambridge University Press, 20091 (2009) 
[2] N. Austern et al., Phys. Rep. 154, 125 (1987)

[3] G.H. Rawitscher, Phys. Rev. C 9, 2210 (1974)

[4] M. Yahiro, Y. Iseri, H. Kameyama, M. Kamimura, M. Kawai, Prog. Theor. Phys. Suppl. 89, 32 (1986)

[5] P. Chau Huu-Tai, Jour. of Phys.: Conf. Series 312, 082018 (2011)

[6] R. Crespo, A. Deltuva, A.M. Moro, Phys. Rev. C 83, 044622 (2011)

[7] A.M. Moro, R. Crespo, Phys. Rev. C 85, 054613 (2012)

[8] N.C. Summers et al., Phys. Rev. C 74, 014606 (2006)

[9] R. de Diego et al., Phys.Rev. C 89, 064609 (2014)

[10] N.C. Summers, F.M. Nunes, Phys. Rev. C 76, 014611 (2007)

[11] A.M. Moro, J.A. Lay, Phys. Rev. Lett. 109, 232502 (2012)

[12] T. Matsumoto et al., Nucl. Phys. A 738, 471 (2004)

[13] M. Rodríguez-Gallardo et al., Phys. Rev. C 77, 064609 (2008)

[14] M. Cubero et al., Phys. Rev. Lett. 109, 262701 (2012)

[15] J.P. Fernández-García et al., Phys. Rev. Lett. 110, 142701 (2013)

[16] L.D. Faddeev, Zh. Eksp. Teor. Fiz. 39, 1459 (1960), [Sov. Phys. JETP 12, $1014(1961)]$

[17] A. Deltuva et al., Phys. Rev. C 76, 064602 (2007)

[18] N.J. Upadhyay, A. Deltuva, F.M. Nunes, Phys. Rev. C 85, 054621 (2012)

[19] N. Matsuoka et al., Nucl. Phys. A 391, 357 (1982)

[20] N. Austern, C.M. Vincent, Phys. Rev. C 23, 1847 (1981)

[21] M. Ichimura, N. Austern, C.M. Vincent, Phys. Rev. C 32, 431 (1985)

[22] G. Potel, F. Nunes, I. Thompson, to be published in Phys. Rev. C, arXiv:1508.04822 (2015)

[23] B.V. Carlson, R. Capote, M. Sin, arXiv:1508.01466 (2015)

[24] J. Lei, A.M. Moro, submitted to Phys. Rev. C (2015) 